\title{
OPEN ISSUES ON THE EAS METHOD AND MESH DISTORTION INSENSITIVE LOCKING-FREE LOW-ORDER UNSYMMETRIC EAS ELEMENTS
}

\author{
Robin Pfefferkorn ${ }^{1 *}$ and Peter Betsch ${ }^{1}$ \\ ${ }^{1}$ Karlsruhe Institute of Technology (KIT) - Institute of Mechanics, \\ Otto-Amann-Platz 9, 76131 Karlsruhe Germany, \\ robin.pfefferkorn@kit.edu, peter.betsch@kit.edu,www.ifm.kit.edu
}

Key words: FEM, mixed finite elements, enhanced assumed strains, Petrov-Galerkin, unsymmetric FEM

\begin{abstract}
One of the most popular mixed finite elements is the enhanced assumed strain (EAS) approach. However, despite numerous advantages there are still some open issues. Three of the most important, namely robustness in nonlinear simulations, hourglassing instabilities and sensitivity to mesh distortion, are discussed in the present contribution. Furthermore, we propose a novel Petrov-Galerkin based EAS method. It is shown that three conditions have to be fulfilled to construct elements that are exact for a specific displacement mode regardless of mesh distortion. The so constructed novel element is lockingfree, exact for bending problems, insensitive to mesh distortion and has improved coarse mesh accuracy.
\end{abstract}

\section{INTRODUCTION}

In the early days of the finite element method (FEM) it was soon discovered that low-order displacement based elements cannot be used efficiently due to severe locking, which denotes numerically induced too stiff behavior compared to "correct" results. A plethora of remedies has subsequently been developed to circumvent this issue. The two main groups are reduced integration with stabilization in explicit simulations and mixed finite elements ${ }^{1}$ in implicit simulations, which are the focus of the present work.

One of the most popular mixed finite elements is the enhanced assumed strain (EAS) approach which is based on a $\mathrm{Hu}$-Washizu functional and was first proposed by Simo and Rifai [2] for linear and Simo and Armero [3] for nonlinear problems, respectively. The EAS approach is a mathematically sound justification for the earlier introduced popular incompatible mode models [4]. Among the many advantages of the method the probably most important one is its strain-driven format which facilitates simple implementation of complex nonlinear material models. This makes EAS elements especially interesting compared to assumed stress (AS) [5,6] which work extremely well in linear problems but require inverse stress-strain relations that rarely exist for nonlinear material models (see e.g. [7, 8]).

Despite all benefits and wide application in practical engineering simulations there are still some open issues and possibilities for improvement of EAS elements. Three of which are discussed in the present work. The first topic concerns robustness in nonlinear simulations by which we denote the size of applicable load steps and number of Newton-Raphson (NR) iterations necessary to obtain convergence. In

\footnotetext{
${ }^{1}$ We denote with a mixed approach any problem from which fields may be removed without loosing the well-definedness of the problem (cf. [1]).
} 
comparison to the AS method, the robustness of EAS elements is poor. This topic is dealt with in depth in the work of Pfefferkorn et al. [8]. Second, spurious hourglassing instabilities still occur in case of elasto-plastic simulations as summarized e.g. by Hille et al. [9]. Finally, EAS elements are sensitive to mesh distortion which is in accordance with MacNeal's theorem [10]. The latter two issues are in fact not only a problem of EAS elements but concern a wide range of other mixed approaches.

The purpose of the present work is twofold. First, the three open issues mentioned above will be explained in more detail including some recent progress. Second, an unsymmetric (i.e. Petrov-Galerkin) EAS method is proposed in order to overcome the limitations by MacNeal's theorem and tackle the third open issue. The key idea is to use a Petrov-Galerkin ansatz in the sense of the unsymmetric FEM method which was proposed by Rajendran and Liew [11] for displacement based finite elements. A comprehensive overview of the method can be found in [12] and [13]. For the novel unsymmetric EAS method we derive three conditions that have to be fulfilled in order to obtain exact solutions for specific displacement modes. Satisfying these conditions for AS stress modes, leads to a locking-free finite element that is mesh-distortion insensitive. In order to facilitate this, the actual choice of ansatz functions for the novel EAS element is closely related to the approach of Huang et al. [14]. However, in contrast to aforementioned reference the present framework is much more generally applicable and can for instance easily be extended to 3D problems.

The present contribution is divided into five sections. Section 2 covers details of the three open issues. In Section 3 the new unsymmetric EAS approach is proposed starting with the three conditions that have to be fulfilled for exact FE solutions in Section 3.1. Afterwards, Section 3.2 covers the ansatz functions used for the novel element. Numerical investigations comparing the present approach to elements follow in Section 2 OPEN ISSUES ON 2.1 Robustness in Newton-Raphson iterations
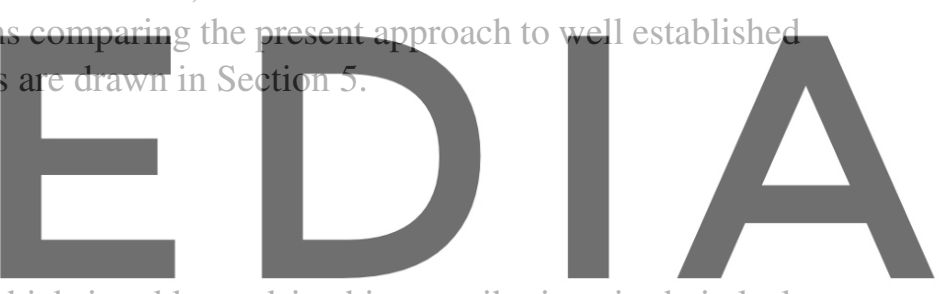

The first open issue concerning EAS elements, which is addressed in this contribution, is their lack

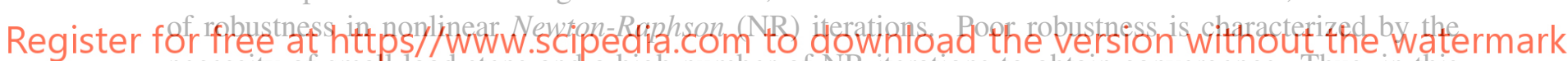
necessity of small load steps and a high number of NR-iterations to obtain convergence. Thus, in this sense, robustness also implies numerical efficiency, since less costly matrix factorizations are necessary. Robustness is thoroughly addressed in the recent work by Pfefferkorn et al. [8].

In the present work, we consider the simple clamped beam example ${ }^{2}$ shown in Fig. 1 for a detailed examination of the phenomenon. Comparison of the required number of NR-iterations $n_{\mathrm{NR}}$ for the AS element Q1/S5 (see e.g. [7]) and the standard nonlinear EAS element Q1/E4 [3] reveals the poor behavior of the latter in terms of robustness.

A simple remedy for that behavior is based on the mixed integration point (MIP) method (see Magisano et al. [15]), which is extended to EAS elements in [8]. The so modified element with increased robustness is denoted Q1/E4-MIP. In case of the St.Venant-Kirchhoff material this element exhibits the same robust results as Q1/S5 in this example. However, Q1/E4-MIP's robustness is still inferior to Q1/S5's for the Neo-Hookean material model and does not even converge in some cases. Finding a robust EAS element for nonlinear material models is thus the first challenge for EAS element development in the future.

\footnotetext{
${ }^{2}$ A thorough description of the example is given in Pfefferkorn et al. [8], where the present results are taken from.
} 


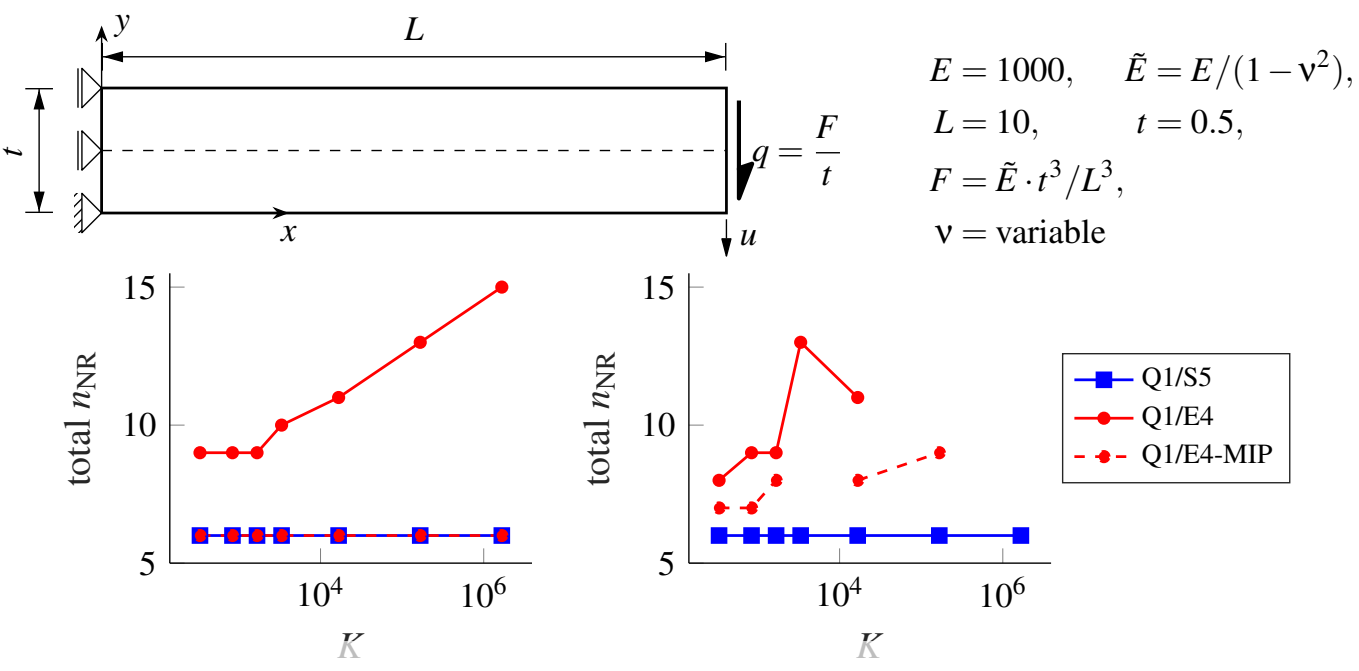

Figure 1: Clamped beam example to examine robustness. Problem setup with geometry and boundary conditions (top). Required number of NR-iterations $n_{\mathrm{NR}}$ in dependence of the bulk modulus $K=E /(3-6 \mathrm{v})$ with one load step for St. Venant-Kirchhoff (bottom left) and a Neo-Hookean (bottom right) material (see [8]) .

\subsection{Hourglass instabilities}

The second open issue are spurious hourglass instablities of EAS elements in nonlinear simulations which are already mentioned in $\mathrm{t}$ work of Wriggers and Ree Q1/E4 [3] in case of a hy fix to overcome this problem in hyperelasticity. T used Witson-Modes as ansatz for the enhanced field

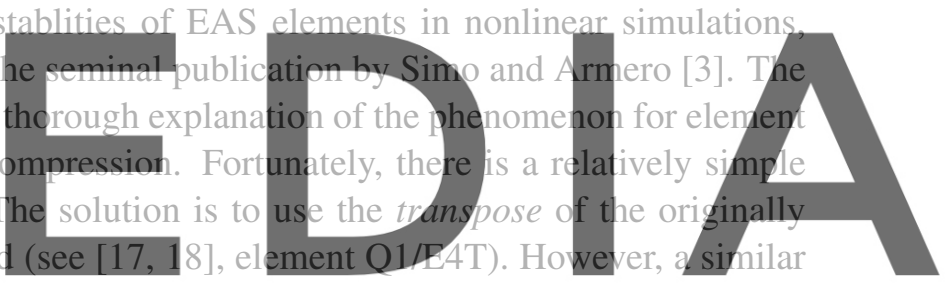
hourglassing phenomenon occurs in elasto-plastic simulations under tension as shown in Fig. 2. It

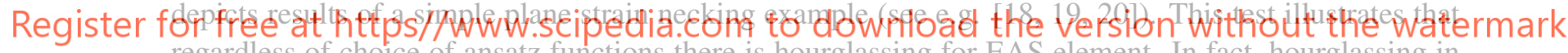
regardless of choice of ansatz functions there is hourglassing for EAS element. In fact, hourglassing in elasto-plastic simulations is not only a problem of EAS elements but concerns other mixed elements such as the one by Armero [19] as well. This is shown in detail in the work of Hille et al. [9].

So far, to the best knowledge of the authors, the only "solutions" to this problem require artificial stabilization terms (see e.g. for EAS elements $[18,21]$ ) or lead to elements that lock (see e.g. [20]) and are therefore not satisfactory. Finding an hourglassing-free EAS element, that does not lock, is thus the second open issue in this overview.

\subsection{Sensitivity to mesh distortion}

The final issue concerns sensitivity of EAS elements to mesh distortion. Once more a simple example (see e.g. [2, 22]) shown in Fig. 3 allows to investigate this problem. The linear elastic clamped beam is subjected to a pure bending moment and meshed with two elements. Parameter $s$ controls the degree of distortion of the specimen. Both the AS element Q1/S5 [5] and the EAS element Q1/E4 [2] are able to give the analytic result $\delta=1$ in case of a rectangular mesh $(s=0)$. However, in case of distorted meshes the performance drops substantially. 

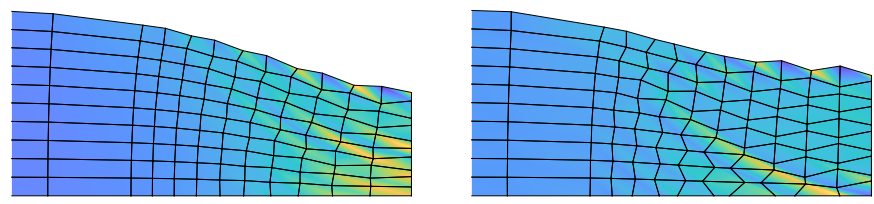

Figure 2: Hourglassing patterns in a 2D elasto-plastic necking simulation. Results for Q1/E4 (left) and Q1/E4T (right) [20].
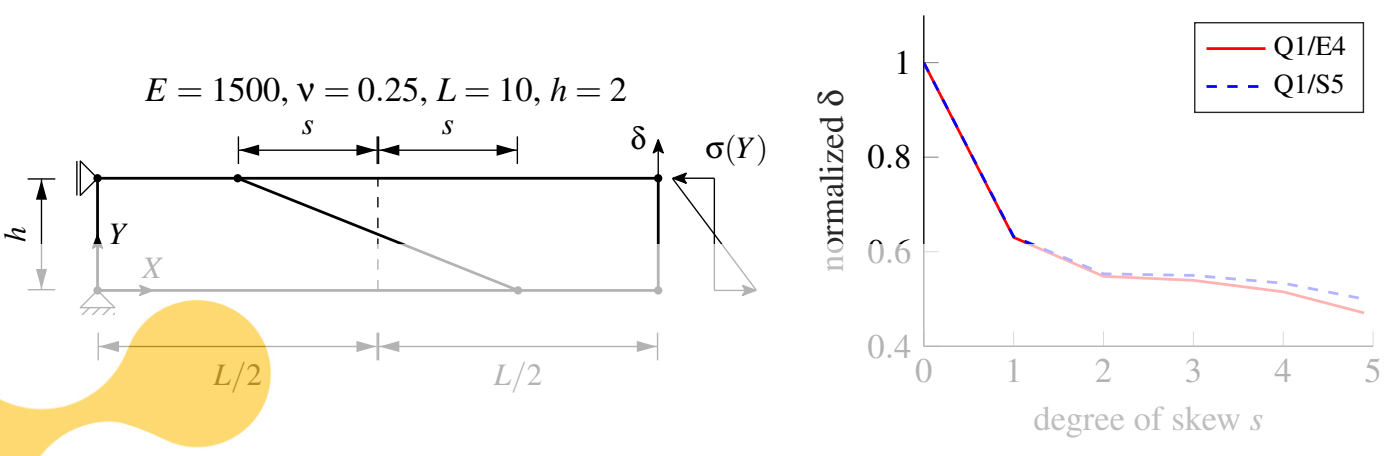

Figure 3: Mesh distortion test (2D). Setup (left) and normalized displacement in dependence of distortion $s$ (right).

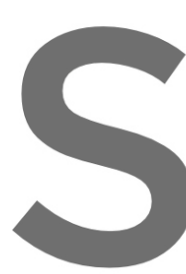

This issue is not only

that has a symmetric stifin

been proven by MacNeal

pass the patch test and $\mathrm{b}$

unsymmetric. Thus, every approach based on the

provements which is in accordance with progress
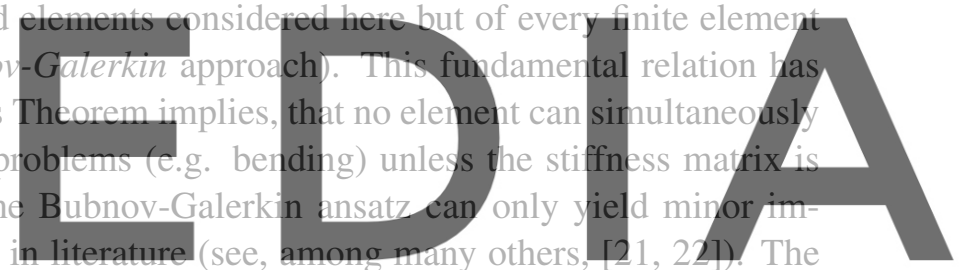

only way to circumvent this issue is to make the element's stiffness matrix unsymmetric by using e.g. a

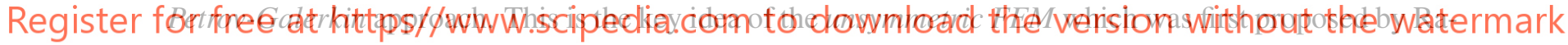
jendran and Liew [11]. However, for low-order elements it is not straightforward to find suitable ansatz spaces. This leads to either extremely complicated ansatz functions, which include material parameters $[13,23]$, or elements that require many internal degrees of freedom and are often not applicable in 3D problems [14].

The remainder of this work is about a way to construct low-order elements on the basis of the EAS framework, which do not require material parameters for their ansatz spaces, are straightforward to construct and are insensitive to mesh distortion.

\section{UNSYMMETRIC EAS ELEMENTS}

\subsection{Conditions for mesh distortion insensitive EAS elements}

Before we propose the actual unsymmetric EAS element in Section 3.2 we derive in this section three conditions which have to be fulfilled in order for an element to be exact for a specific displacement mode ${ }^{3}$ $\mathbf{u}^{*}$. Corresponding to $\mathbf{u}^{*}$, the usual relations determine the strains $\varepsilon^{*}=\nabla_{\mathbf{x}}^{s} \mathbf{u}^{*}$, stresses $\sigma^{*}=\mathbb{C}: \varepsilon^{*}$, and

\footnotetext{
${ }^{3}$ Usually a linear combination of polynomial expressions.
} 


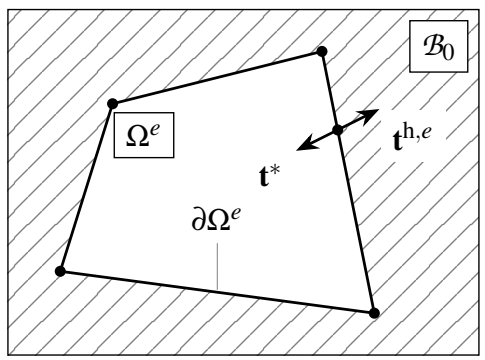

Figure 4: Single finite element $\Omega^{e}$ embedded in linear elastic continuum.

tractions $\mathbf{t}^{*}=\sigma^{*} \mathbf{n}^{*}$ where $\mathbb{C}$ is the elasticity tensor.

To that end we consider in analogy to the method in [10] a single finite element $\Omega^{e}$ with boundary $\partial \Omega^{e}$ embedded into a linear elastic continuum $\mathcal{B}_{0}$ (see Fig. 4). The weak form of a single EAS element [2] is given by

$$
\begin{aligned}
& \int_{\Omega^{e}} \nabla_{\mathbf{x}}^{S} \mathbf{v}^{\mathrm{h}, e}: \sigma^{\mathrm{h}, e} \mathrm{~d} V+\Pi_{\mathrm{ext}}\left(\mathbf{v}^{\mathrm{h}, e}\right)=0, \\
& \int_{\Omega^{e}} \tilde{\mathrm{e}}^{\mathrm{h}, e}: \sigma^{\mathrm{h}, e} \mathrm{~d} V=0,
\end{aligned}
$$

where $\mathbf{v}^{\mathrm{h}, e}$ and $\tilde{\mathrm{e}}^{\mathrm{h}, e}$ are the test functions of the displacements and enhanced strains, respectively. The discrete constitutive stro tions for the displacement is given by
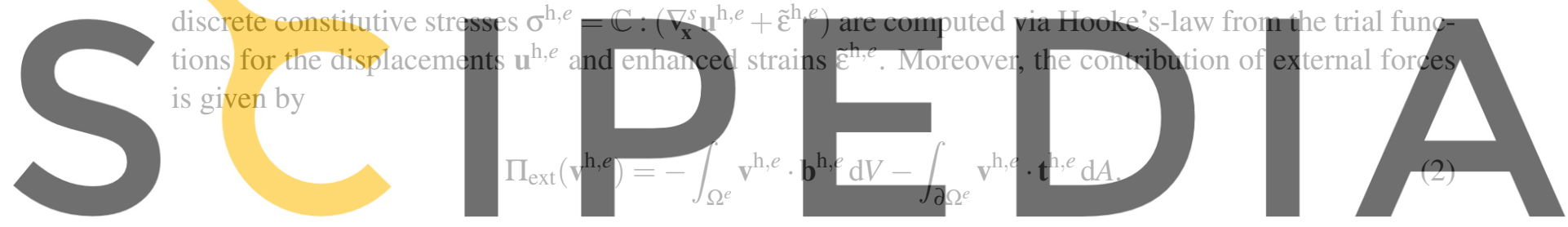

Instead of the usual approach of selecting $\mathbf{v}^{\mathrm{h}, e}$ and $\mathbf{u}^{\mathrm{h}, e}$ as well as $\tilde{\mathrm{e}}^{\mathrm{h}, e}$ and $\tilde{\varepsilon}^{\mathrm{h}, e}$ form the same ansatz spaces

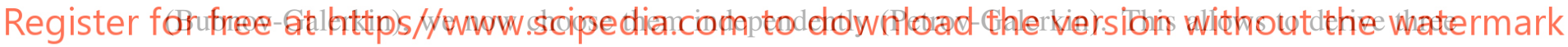
conditions which have to be fulfilled in order to obtain an exact FE solution.

The first condition foliows from the fact, that we oniy consider a single eiement. In order to get correct solutions in case of larger FE-meshes it has to be ensured that the inter-element continuity is maintained. That is the case if nodal equilibrium is exactly fulfilled regardless of the element's geometry. The test functions for the displacements have to be chosen appropriately in order to fulfill this condition, which is a fundamental result of FE-design (see e.g. [1]).

The other two conditions can be derived by rearranging (1). Under the assumption that the discrete external forces $\mathbf{b}^{\mathrm{h}, e}$ and $\mathbf{t}^{\mathrm{h}, e}$ can exactly represent the continuum counterparts $\mathbf{b}^{*}=-\operatorname{div} \sigma^{*}$ and $\mathbf{t}^{*}=\sigma^{*} \mathbf{n}^{*}$, we can recast (2) by virtue of the divergence theorem in the form

$$
\Pi_{\text {ext }}\left(\mathbf{v}^{\mathrm{h}, e}\right)=-\int_{\Omega^{e}} \mathbf{v}^{\mathrm{h}, e} \cdot \mathbf{b}^{*} \mathrm{~d} V-\int_{\partial \Omega^{e}} \mathbf{v}^{\mathrm{h}, e} \cdot \mathbf{t}^{*} \mathrm{~d} A=-\int_{\Omega^{e}} \nabla_{\mathbf{x}}^{s} \mathbf{v}^{\mathrm{h}, e}: \sigma^{*} \mathrm{~d} V .
$$

Inserting this result into (1a) yields

$$
\int_{\Omega^{e}} \nabla_{\mathbf{x}}^{s} \mathbf{v}^{\mathrm{h}, e}:\left(\sigma^{\mathrm{h}, e}-\sigma^{*}\right) \mathrm{d} V=0 .
$$


Since $\mathbf{v}^{\mathrm{h}, e}$ is not arbitrary due to the first condition discussed above, equation (4) can only be fulfilled in general if $\sigma^{\mathrm{h}, e}=\sigma^{*}$, which has to be ensured by the ansatz spaces for $\mathbf{u}^{\mathrm{h}, e}$ and $\tilde{\varepsilon}^{\mathrm{h}, e}$. A final condition follows from inserting $\sigma^{\mathrm{h}, e}=\sigma^{*}$ into (1b). This yields

$$
\int_{\Omega^{e}} \tilde{\mathbf{e}}^{\mathrm{h}, e}: \sigma^{*} \mathrm{~d} V=0,
$$

which implies that the test function for the enhanced strains $\tilde{\mathbf{e}}^{\mathrm{h}, e}$ must be $L_{2}$-orthogonal to $\sigma^{*}$. This final condition is essentially an extension of the orthogonality condition which is necessary for the classical EAS element in order to fulfill the patch test [2].

Summarizing these considerations we have three conditions, which have to be fulfilled in order to construct an unsymmetric EAS element that is exact for a chosen mode $\mathbf{u}^{*}$ :

C1 The test functions $\mathbf{v}^{\mathrm{h}, e}$ must fulfill nodal equilibrium regardless of element geometry and neighboring elements.

C2 The ansatz for the discrete stress $\sigma^{\mathrm{h}, e}$, which is computed from $\mathbf{u}^{\mathrm{h}, e}$ and $\tilde{\varepsilon}^{\mathrm{h}, e}$, must include the stresses $\sigma^{*}$

C3 The test function for the enhanced strains $\tilde{\mathbf{e}}^{\mathrm{h}, e}$ must be $L_{2}$-orthogonal to $\sigma^{*}$ (see (5)).

\subsection{Element design}

\subsubsection{Choice of analytic modes}

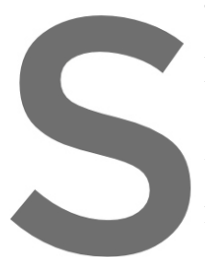

Before choosing the antatispaces f

Before choosing the ansat

strain field, it is necessary

we only consider four-no

freedom in the $2 \mathrm{D}$ case an

As pointed out by Cen et al. [231 there is unfort
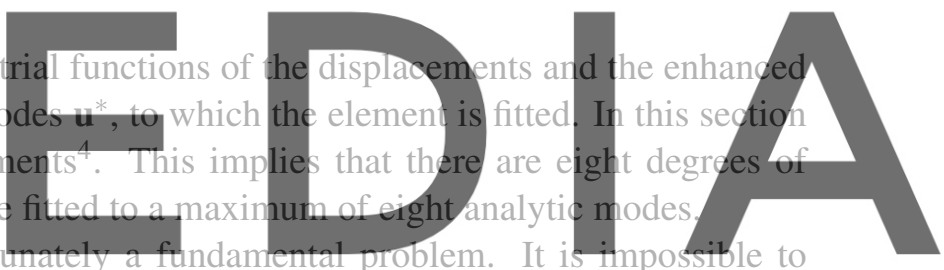

incorporate fully quadratic displacements since that requires twelve displacement modes. Furthermore,

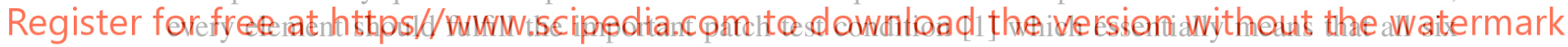

linear displacement modes must be included in the element's space. Thus, of the eight modes available,

only two can be chosen freely instead of the six necessary for fully quadratic solutions. These modes must be chosen with care in order to maximize the element's performance.

In many engineering applications, bending problems are of the utmost importance. This is why we propose to use the assumed stress modes introduced by Pian and Sumihara [5] as analytic modes. These five modes include, apart from the two pure bending modes around the element axes, also the three constant stress patch test modes. Together with the three rigid body modes this adds up to the eight available ones. The only change made to the modes from [5] is using a skew coordinate frame as proposed by Wisniewski and Turska [24]. Ultimately this yields the analytic modes

$$
\sigma^{*}=\mathbf{J}_{0} \hat{\sigma}^{*}(\bar{\xi}) \mathbf{J}_{0}^{\mathrm{T}}, \quad \hat{\sigma}_{v}^{*}=\left[\begin{array}{ccccc}
1 & 0 & 0 & \bar{\xi}_{2} & 0 \\
0 & 1 & 0 & 0 & \bar{\xi}_{1} \\
0 & 0 & 1 & 0 & 0
\end{array}\right]
$$

\footnotetext{
${ }^{4} 3 \mathrm{D}$ elements are considered in the numerical investigation in Section 4.
} 


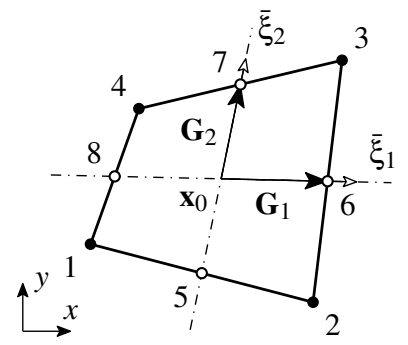

Figure 5: Higher order parent element with internal nodes (hollow dots), standard nodes (full dots) and skew coordinate system.

where $\mathbf{J}_{0}$ is the Jacobian of the isoparametric map $\mathbf{x}^{\mathrm{h}, e}(\xi)=\sum_{I} N_{I}(\xi) \mathbf{x}_{I}$ evaluated at the element's centroid, $\xi$ denotes the coordinates of the reference element and $N_{I}$ are the standard bi-linear Lagrangian shape functions. Moreover,

$$
\bar{\xi}=\mathbf{J}_{0}^{-1}\left(\mathbf{x}^{\mathrm{h}, e}-\mathbf{x}_{0}\right)
$$

are the skew coordinates where $\mathbf{x}_{0}=\mathbf{x}^{\mathrm{h}, e}(\xi=\mathbf{0})$ is the element's centroid.

\subsubsection{Ansatz functions}

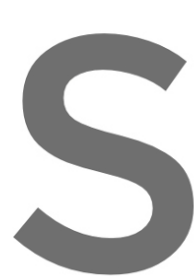

The construction of ans

mode approach by Huang et al.

method is easily extended to 3D

serendipity parent elemen

The key idea for the ansatz functions is to consider a

5. On top of the usual four nodes of the quadrilateral element (full
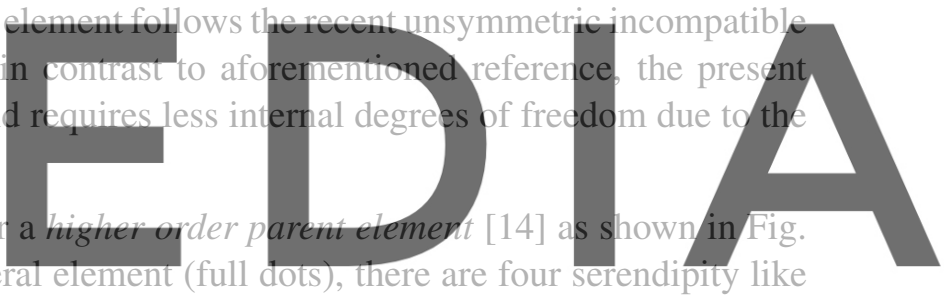

internal nodes (hollow dots) that are only used on element level. Starting from that basis we now propose

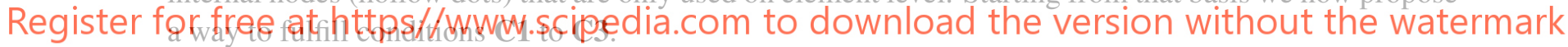

In order to fulfill condition $\mathbf{C} \mathbb{1}$ it is necessary for the test functions of the displacements to enable nodal

equilibrium. This is exactily what the isoparametric concept was developed for (see e.g. [1]). Thus, we use the usual bi-linear Lagrangian shape functions $N_{I}=\frac{1}{4}\left(1+\xi_{I} \xi\right)\left(1+\eta_{I} \eta\right)$ to approximate the test functions $\mathbf{v}^{\mathrm{h}, e}$.

Next we consider condition C3. To fulfill it we start with the serendipity modes $N_{5}$ to $N_{8}$ associated with the internal nodes. Their derivatives are used to approximate intermediate enhanced strains given by

$$
{ }^{\mathrm{i}} \tilde{\mathbf{e}}^{\mathrm{h}, e}=\frac{1}{j^{\mathrm{h}, e}} \mathbf{J}_{0}^{-\mathrm{T}} \operatorname{sym}\left(\sum_{I=5}^{8} \alpha_{I} \otimes \nabla_{\xi} \hat{N}_{I}\right) \mathbf{J}_{0}^{-1} .
$$

Unfortunately, these modes do not fulfill (5) in general. However, it is possible to ensure (5) by an orthogonalization procedure. First, the special approximations of (6) and (8) allow to simplify (5) in the form

$$
\int_{\Omega^{e}} \tilde{\mathbf{e}}^{\mathrm{h}, e}: \sigma^{*} \mathrm{~d} V=\int_{\hat{\Omega}} \mathrm{i} \hat{\mathbf{e}}_{v}^{\mathrm{h}, e} \cdot \hat{\sigma}_{v}^{*} \mathrm{~d} \hat{\Omega}=0 .
$$


The next step is to apply Gram-Schmidt orthogonalization to the enhanced strains ${ }^{5}$. This yields the actual ansatz for the enhanced strains in the form

$$
\hat{\mathbf{e}}_{v, I}^{\mathrm{h}, e}={ }^{\mathrm{i}} \hat{\mathbf{e}}_{v, I}^{\mathrm{h}, e}-\sum_{k=1}^{5} \frac{\int_{\hat{\Omega}}^{\mathrm{i}} \hat{\mathbf{e}}_{v, I}^{\mathrm{h}, e} \cdot \hat{\sigma}_{v, k}^{*} \mathrm{~d} \hat{\Omega}}{\int_{\hat{\Omega}} \hat{\sigma}_{v, k}^{*} \cdot \hat{\sigma}_{v, k}^{*} \mathrm{~d} \hat{\Omega}} \hat{\boldsymbol{\sigma}}_{v, k}^{*}, \quad \tilde{\mathbf{e}}^{\mathrm{h}, e}=\frac{1}{j^{\mathrm{h}, e}} \mathbf{J}_{0}^{-\mathrm{T}} \hat{\mathbf{e}}^{\mathrm{h}, e} \mathbf{J}_{0}^{-1} .
$$

The final condition, that needs to be fulfilled, is condition C2. It requires that linear stresses in the physical space (see (6)) can be exactly represented. Corresponding displacements need thus to be fully quadratic. For this purpose, isoparametric ansatz functions are not suitable since they cannot represent fully quadratic polynomials in the physical space in case of distorted meshes due to the rational-valued transformation. However, metric shape functions, which were proposed for unsymmetric elements by Rajendran and Liew [11], allow fully quadratic polynomials in the physical space regardless of mesh distortion. For the serendipity type parent element we compute these functions in the skew-coordinate frame as proposed by Xie et al. [13]. Using the skew frame is necessary in order to obtain isotropic and frame-invariant ansatz functions. Note that the linear transformation (7) preserves the fully quadratic polynomials in the physical space.

With these ansatz functions at hand we now have an EAS element that is insensitive to mesh distortion and exact in pure bending problems. This is shown in detail in the numerical investigation in Section 4. Furthermore, the present approach can easily be applied with the very same steps in 3D simulations, which is in contrast to the work of Huang et al. [14].
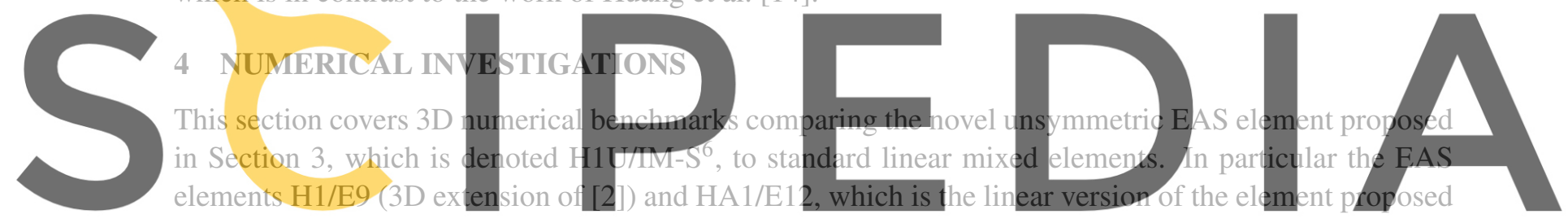

in [22], are considered. Furthermore, we use the AS element with 18 stress modes H1/S18 by Pian and

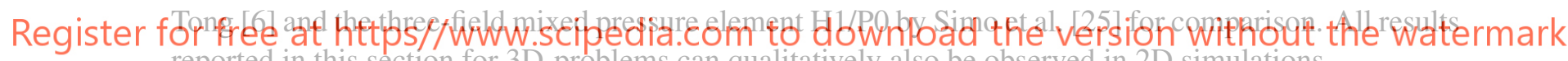
reported in this section for 3D-problems can qualitatively also be observed in 2D simulations.

\subsection{Patch test}

The first example is the classical patch test in the form described in [22]. All elements tested fulfill this basic test. For the present element this follows from the choice of analytic modes (6). Since the AS modes include constant stress modes, element H1U/IM-S fulfills the patch test by construction.

\subsection{Isotropy and invariance test}

This test, which is taken from [13], can be used to test if the element satisfies two fundamental conditions, namely isotropy and frame invariance. The geometry and boundary conditions of the test are shown in Fig. 6. The first condition concerns isotropy which denotes if the element is invariant to orientation of the local reference element axes $\xi \eta \zeta$ in relation to the local system $\bar{X} \bar{Y} \bar{Z}$. It can be checked by rearranging the local node numbering EDOF. Second, frame invariance is given if the element is invariant with respect

\footnotetext{
${ }^{5}$ The modes of $\hat{\sigma}^{*}$ are in general not orthogonal to each other and need to be orthogonalized in a first step.

${ }^{6} \mathrm{IM}$ stands for incompatible mode and $\mathrm{S}$ denotes the serendipity type parent element.
} 


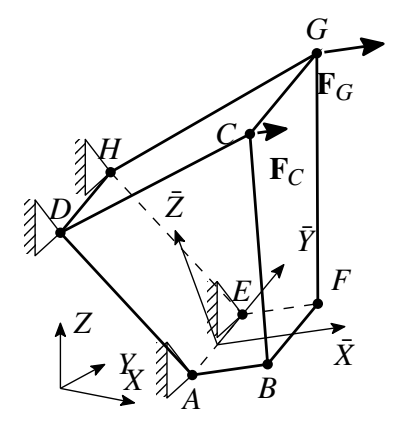

Nodes and force:

$$
A=(0,-0.75,0)
$$

$B=(1,-0.75,0)$

$E=(0,0.75,0)$

$C=(2,-1,3)$

$F=(1,0.75,0)$

$D=(-1,-0.75,2)$

$\mathbf{F}_{C}=[100,0]^{\mathrm{T}}$
$G=(2,1,3)$

$H=(-1,0.75,2)$

$\mathbf{F}_{G}=[200,0,0]^{\mathrm{T}}$
Element node-numberings:

$\mathrm{EDOF}_{1}=[A, B, C, D, E, F, G, H]$

$\mathrm{EDOF}_{2}=[F, B, C, G, E, A, D, H]$

$\mathrm{EDOF}_{3}=[A, D, C, B, E, H, G, F]$

Figure 6: Setup of isotropy and invariance test in 3D.
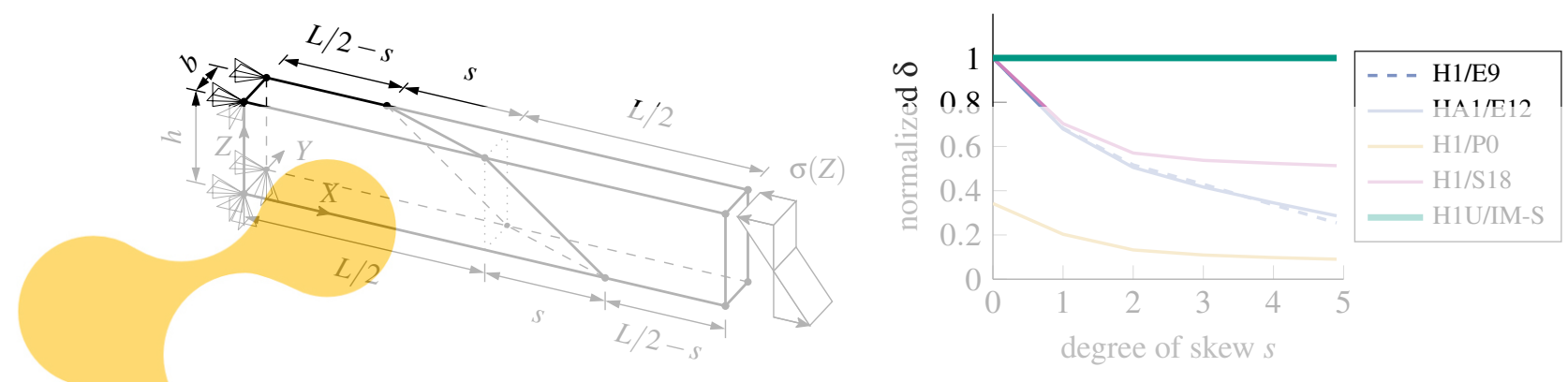

Figure 7: Mesh distortion test (3D). Setup (left) and normalized displacement in dependence of distortion $s$ (right).

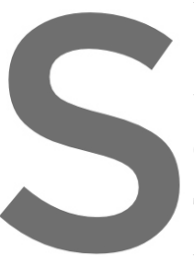

to rotation of the global coordinate system XYZ in re
checked by changing the angle between the global co
The results are evaluated by measuring the displace rotation nor with node-numbering, all elements tester
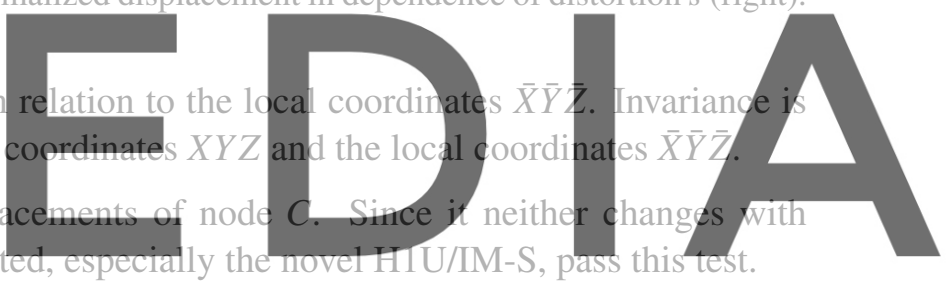

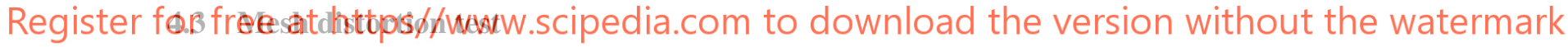

This test has already been used in Section 2.3 to demonstrate MacNeal's Theorem [10]. Here, we consider a 3D equivalent with dimensions $L=10, h=2$ and $b=1$ shown in Fig. 7. Again, displacement $\delta$ is normalized using the analytic result and plotted in dependence of the distortion $s$ on the right of Fig. 7. Since coincident nodes $(s=5)$ are not allowed in order to compute the metric shape functions, this state had to be excluded and the maximum distortion is set to $s=4.9$.

As predicted by MacNeal's Theorem, none of the Bubnov-Galerkin mixed finite elements is able to predict exact displacements with the exemption of no distortion. The performance drops quickly with higher values of $s$. Of the symmetric elements, H1/P0 exhibits the worst results since shear locking is not alleviated. The clearly best results are obtained with element H1U/IM-S. It shows perfect results regardless of mesh distortion, since it was designed for this pure bending problem. This is in line with other low-order unsymmetric finite elements (see e.g. [13, 14, 23]). However, in contrast to [13, 23], the element here does not need material parameters for its ansatz functions and can be extended to $3 \mathrm{D}$ problems, which is not obvious with the element proposed in [14]. 

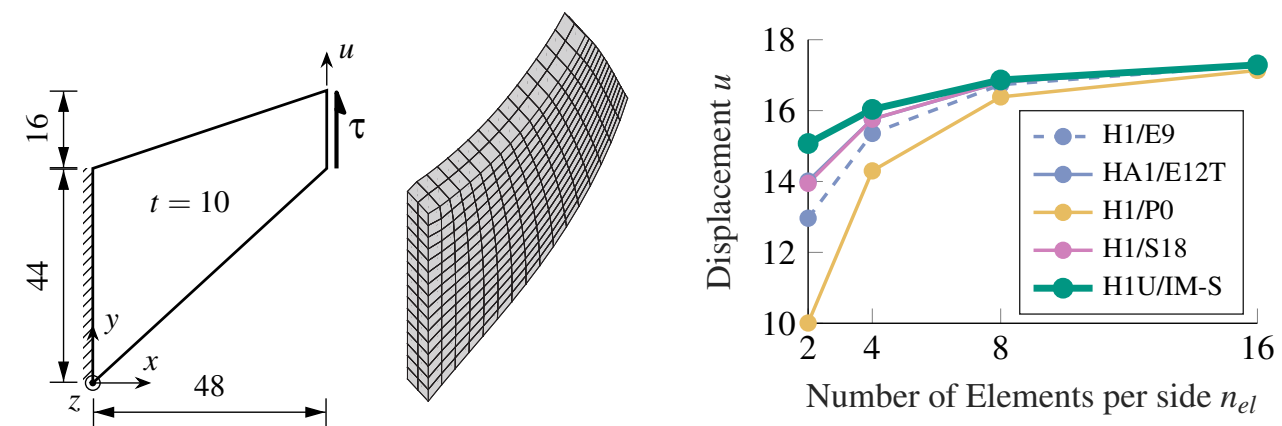

Figure 8: Cooks membrane. Setup (left), displacement figure (middle) and displacement with mesh refinement (right).

\subsection{Cooks membrane}

The final test is the famous Cook's membrane benchmark which is included here as described in detail in [22]. Fig. 8 shows the setup and results of the test. To consider nearly incompressible material behavior the material parameters are chosen as $E=2261.2$ and $v=0.49547$.

Due to the complex deformation state in this example, it is not possible to obtain exact solutions with any finite element. However, the plot in Fig. 8 shows that the novel element H1U/IM-S exhibits the best performance especially for coarse meshes. In case of fine meshes the difference becomes smaller but the

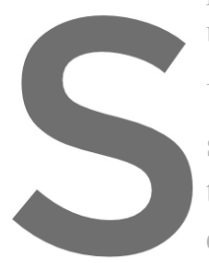
unsymmetric approach Unfortunately, the improv solving the linear equation that the unsymmetric element needsup to 4.74 tim ements (more than 114,000 DOFs). Furthermore, t.
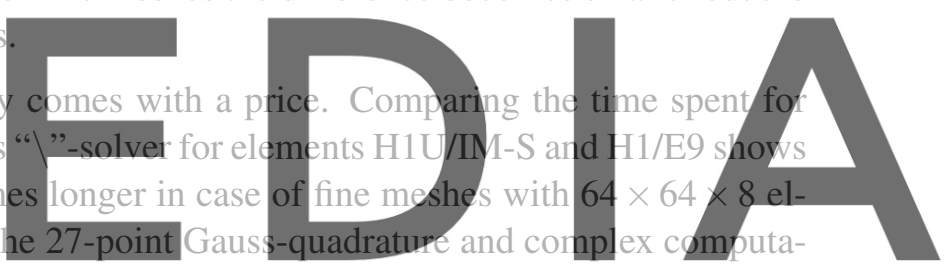

tion of shape functions further increase numerical cost. However, since H1U/IM-S allows using coarser

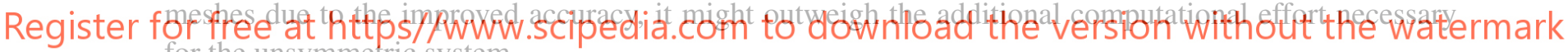
for the unsymmetric system.

\section{CONCLUSION}

In the present work we proposed a novel linear low-order unsymmetric finite element based on the EAS framework. Starting from the weak form for EAS elements [2] three conditions have been derived, that allow to construct an element which is exact for an arbitrary displacement mode.

On this basis we developed a EAS element, which is exact for the classical assumed stress modes [5, 6]. The novel element is free from locking, exact for pure bending problems, insensitive to mesh distortion and has improved coarse mesh accuracy. In contrast to most existing low-order unsymmetric elements it does not require material parameters to compute the ansatz functions and it is easily applicable in 3D simulations.

Drawbacks of the element are related to computational cost. First, the unsymmetric FEM always requires a more costly linear solver. Second, the higher order internal serendipity modes require many enhanced degrees of freedom (36 in 3D) and a higher order Gauss-quadrature, which makes the element routine itself expensive. 
In future works, the first goal should be to reduce the number of internal degrees of freedom as well as the order of Gauss-quadrature. Secondly, an extension of the current framework to nonlinear problems would be of the utmost interest.

\section{REFERENCES}

[1] Zienkiewicz, O. C., Taylor, R. L., and Zhu, J. The Finite Element Method. Vol. 1: Its Basis and Fundamentals. Sixth. Amsterdam: Elsevier Butterworth-Heinemann, (2010).

[2] Simo, J. C. and Rifai, M. S. A Class of Mixed Assumed Strain Methods and the Method of Incompatible Modes. Int. J. Numer. Meth. Engng., (1990) 29(8): 1595-1638.

[3] Simo, J. C. and Armero, F. Geometrically Non-Linear Enhanced Strain Mixed Methods and the Method of Incompatible Modes. Int. J. Numer. Meth. Engng., (1992) 33(7): 1413-1449.

[4] Taylor, R. L., Beresford, P. J., and Wilson, E. L. A Non-Conforming Element for Stress Analysis. Int. J. Numer. Meth. Engng., (1976) 10(6): 1211-1219.

[5] Pian, T. H. H. and Sumihara, K. Rational Approach for Assumed Stress Finite Elements. Int. J. Numer. Meth. Engng., (1984) 20(9): 1685-1695.

[6] Pian, T. H. H. and Tong, P. Relations between Incompatible Displacement Model and Hybrid Stress Model. Int. J. Numer. Meth. Engng., (1986) 22(1): 173-181.

[7] Viebahn, N., Schröder, J., and Wriggers, P. An Extension of Assumed Stress Finite Elements to a General Hyperelastic Framework. Adv. Model. and Simul. in Eng. Sci., (2019) 6(9).

[8] Pfefferkorn, R., Bieber, S., Oesterle, B., Bischoff, M., and Betsch, P. Improving Efficiency and Robustness of EAS Elements for Nonlinear Problems. Int. J. Numer. Meth. Engng., (2020) 1-29. DOI: $10.1002 / \mathrm{nme} .6605$.

[9] Hille, M., Pfefferkorn, R., and Betsch, P. "Locking-Free Mixed Finite Element Methods and Their Spurious Hourglassing Patterns". Current Trends and Open Problems in Computational Mechanics. Springer, (2021), 1-13.

[10] MacNeal, R. H. On the Limits of Finite Element Perfectability. Int. J. Numer. Meth. Engng., (1992) 35(8): 1589-1601.

[11] Rajendran, S. and Liew, K. M. A Novel Unsymmetric 8-Node Plane Element Immune to Mesh Distortion under a Quadratic Displacement Field. Int. J. Numer. Meth. Engng., (2003) 58(11): 1713-1748.

[12] Rajendran, S. A Technique to Develop Mesh-Distortion Immune Finite Elements. Comput. Methods Appl. Mech. Engrg., (2010) 199(17): 1044-1063.

[13] Xie, Q., Sze, K. Y., and Zhou, Y. X. Modified and Trefftz Unsymmetric Finite Element Models. International Journal of Mechanics and Materials in Design, (2016) 12(1): 53-70.

[14] Huang, Y., Huan, Y., and Chen, H. An Incompatible and Unsymmetric Four-Node Quadrilateral Plane Element with High Numerical Performance. Int. J. Numer. Meth. Engng., (2020) 121(15): 3382-3396.

[15] Magisano, D., Leonetti, L., and Garcea, G. How to Improve Efficiency and Robustness of the Newton Method in Geometrically Non-Linear Structural Problem Discretized via DisplacementBased Finite Elements. Comput. Methods Appl. Mech. Engrg., (2017) 313: 986-1005.

[16] Wriggers, P. and Reese, S. A Note on Enhanced Strain Methods for Large Deformations. Comput. Methods Appl. Mech. Engrg., (1996) 135(3-4): 201-209. 
[17] Korelc, J. and Wriggers, P. Consistent Gradient Formulation for a Stable Enhanced Strain Method for Large Deformations. Engineering Computations, (1996) 13(1): 103-123.

[18] Glaser, S. and Armero, F. On the Formulation of Enhanced Strain Finite Elements in Finite Deformations. Engineering Computations, (1997) 14(7): 759-791.

[19] Armero, F. On the Locking and Stability of Finite Elements in Finite Deformation Plane Strain Problems. Computers \& Structures, (2000) 75(3): 261-290.

[20] Pfefferkorn, R. and Betsch, P. Extension of the Enhanced Assumed Strain Method Based on the Structure of Polyconvex Strain-Energy Functions. Int. J. Numer. Meth. Engng., (2020) 121(8): 1695-1737.

[21] Korelc, J., Šolinc, U., and Wriggers, P. An Improved EAS Brick Element for Finite Deformation. Comput. Mech., (2010) 46(4): 641-659.

[22] Pfefferkorn, R. and Betsch, P. On Transformations and Shape Functions for Enhanced Assumed Strain Elements. Int. J. Numer. Meth. Engng., (2019) 120(2): 231-261.

[23] Cen, S., Zhou, P.-L., Li, C.-F., and Wu, C.-J. An Unsymmetric 4-Node, 8-DOF Plane Membrane Element Perfectly Breaking through MacNeal's Theorem. Int. J. Numer. Meth. Engng., (2015) 103(7): 469-500.

[24] Wisniewski, K. and Turska, E. Improved Four-Node Hellinger-Reissner Elements Based on Skew Coordinates. Int. J. Numer. Meth. Engng., (2008) 76(6): 798-836.

[25] Simo, J. C., Taylor, R. L., and Pister, K. S. Variational and Projection Methods for the Volume Constraint in Finite Deformation Elasto-Plasticity. Comput. Methods Appl. Mech. Engrg., (1985) 51(1): 177-208. 